\title{
Cardiomyocyte differentiation: Experience and observations from 2 laboratories
}

V.A. Patten", I. Chabaesele", B. Sishi" and D. van Vuuren"

"Department of Biomedical Sciences, Division of Medical Physiology, Faculty of Medicine and Health Sciences, University of Stellenbosch, Tygerberg, South Africa

"Department of Physiological Sciences, Faculty of Natural Sciences, University of Stellenbosch, Stellenbosch, South Africa

Address for correspondence:

Dr Derick van Vuuren

Department of Biomedical Sciences

University of Stellenbosch

Tygerberg

7505

South Africa

Email:

dvvuuren@sun.ac.za

\section{INTRODUCTION}

The affliction of cardiovascular disease (CVD) is escalating rapidly throughout the world, with the majority of sufferers residing in developing countries. ${ }^{(1)}$ According to the World Health Organisation (WHO), CVD is the leading cause of death globally accounting for an estimated $31 \%$ of all deaths worldwide. (2) Cardiovascular disease also poses a threat to the African continent, with several studies showing the prominence of a variety of cardiovascular afflictions in the African context, including heart failure and systolic dysfunction, ${ }^{(3)}$ ischaemic heart disease $(\mathrm{IHD}){ }^{(4)}$ hypertension and hypertensive heart disease $^{(5)}$ with associated heart failure. ${ }^{(6)}$ Available data from the Medical Research Council of South Africa suggests that the predominance and treatment of common heart conditions in the population such as heart failure, cardiac hypertrophy and diseases of the valves and heart muscle are to a large extent, poorly managed. (7) Duly, it has become a crucial goal for researchers to develop and implement new and improved treatment strategies to protect the heart against disease and injury.

In previous research to date, a number of in vitro cell based models have been used to investigate heart physiology and pathology, with the advantage that cell models provide an ethically acceptable research tool, enabling the targeting of specific cellular processes with relative consistency and reproducibility. ${ }^{(8)}$ In 1976, Kimes and Brandt established an undifferentiated clonal cell line, $\mathrm{H} 9 \mathrm{c} 2$, derived from embryonic

\section{ABSTRACT}

The undifferentiated clonal cell line, $\mathrm{H} 9 \mathrm{c} 2$, derived from left ventricular rat heart tissue, has been extensively used in cardiovascular research. In the present study, 2 independent laboratories aimed to investigate the cells' capacity to differentiate into distinct cardiac-like cells. Undifferentiated $\mathrm{H} 9 \mathrm{c} 2$ cells were supplemented daily for a period of 6 - 12 days, with varying concentrations of retinoic acid (RA) ( $10 \mathrm{nM}, 30 \mathrm{nM}$ and $I \mu M)$, in standard cell culture medium containing either $1 \%$ foetal bovine, or horse serum, in order to stimulate differentiation of the cells into a more cardiac-specific phenotype. Light microscopy confirmed some degree of morphological change associated with differentiation, and a significant increase in oxidative phosphorylation following RA treatment was observed. However, Western blot probing for the cardiac-specific markers Cardiac Troponin T (cTnT) and Myosin Light Chain-2v (MLC2v) indicated little to no differentiation, although immunocytochemistry indicated the presence of cTnT expression. Thus, it was found that the differentiation protocol induced differentiation in some, but not all cells, thereby generating a heterogeneous cell population. Our findings suggest that the $\mathrm{H} 9 \mathrm{c2}$ cell line may display some degree of resistance to differentiation.This should be kept in mind when considering to use this model for cardiovascular research. SAHeart 20 17;14:96-107

left ventricular rat heart tissue. ${ }^{(9)}$ While the cells do not beat and differ morphologically from isolated adult cardiomyocytes, similarities with primary cardiomyocytes have been reported, specifically in terms of the presence and dynamics of L-type calcium channels, a similar cell membrane lectin binding pattern and comparable G-protein composition associated with ligand stimulated intracellular signalling. ${ }^{(10,1)}$ As such, the $\mathrm{H} 9 \mathrm{c} 2$ cell line has been used extensively in research to study the mechanisms of cardiac hypertrophy, ${ }^{(12)}$ oxidative stress, ${ }^{(13,14)}$ ischaemia/ reperfusion (I/R), ${ }^{(15,16)}$ and cardiotoxicity. ${ }^{(17)}$ These cells, however, also have the capacity to adopt features of differentiated skeletal or cardiac muscle upon long term culture with low serum levels, ${ }^{(9,18)}$ and in the case of cardiac specific differentiation, daily treatment with retinoic acid (RA). ${ }^{(19,20)}$

It has previously been reported that $\mathrm{H} 9 \mathrm{c} 2$ cells maintained at confluency for an extended period of time (7 - 10 days), in the presence of relatively low concentrations of serum ( $1 \%)$, as well 
as daily supplementation with RA, exhibit features indicative of cardiac specific differentiation. ${ }^{(19,20)}$ These include an increased expression of cardiac specific proteins such as Myosin Light chain-2v (MLC-2v) and Cardiac Troponin T (cTnT), ${ }^{(19,20)}$ as well as a shift in metabolic preference from primarily glycolytic to oxidative metabolism. ${ }^{(20)}$ Lopashuk, et al. (2010) identified that a switch from glycolysis to oxidative phosphorylation is a fundamental characteristic of differentiated cardiac cells, since cardiomyotubes prefer fatty acids to glucose as a source of fuel. ${ }^{(21)}$

It could be argued that when differentiated, the $\mathrm{H} 9 \mathrm{c} 2$ cells would offer a more closely matched representation of adult cardiomyocytes than when undifferentiated. Menard, et al. (1999) stated that the $\mathrm{H} 9 \mathrm{c} 2$ cells are easy to manipulate, and when differentiated, provide a suitable cardiac cellular model with a maintained cardiac phenotype for a range of investigations commonly challenging to perform on primary cardiomyocytes. ${ }^{(19)}$ It is therefore tempting to pursue differentiation of these cells as a more relevant experimental model than the undifferentiated cardiomyoblast cell line.

This paper will describe the efforts of 2 laboratories, working independently from one another, to differentiate $\mathrm{H} 9 \mathrm{c} 2$ cells. Both laboratories are affiliated with the University of Stellenbosch (South Africa). "Lab I" is situated at the department of Physiological Sciences in the Faculty of Natural Sciences, while "Lab 2" is part of the division of Medical Physiology in the Faculty of Medicine and Health Sciences. Each laboratory independently investigated the capacity of the $\mathrm{H} 9 \mathrm{c} 2$ cell line to differentiate into a more cardiac-specific cell line upon daily treatment with RA and/or reduced serum conditions as described by Pereira, et al. and Menard, et al. respectively. ${ }^{(19,20)}$

Lab I made use of bright field microscopy to investigate morphology, Western blotting to investigate the expression of the cardiac specific marker MLC-2v, and immunocytochemistry to assess the expression of a second cardiac specific marker, cTnT. Oxidative phosphorylation analysis was carried out to establish whether any changes in oxygen consumption had occurred following differentiation. Similarly, following daily RA treatment, Lab 2 attempted to confirm differentiation of the H9c2 cell line using Western blotting techniques to probe for the expression of the cardiac specific markers MLC and CTnT.

Although it was hypothesised that $\mathrm{H} 9 \mathrm{c} 2$ cells would easily differentiate to offer a good representation of cardiac physiology, both laboratories generated data suggesting that the ability of these cells to fully differentiate is somewhat limited. This implies that the $\mathrm{H} 9 \mathrm{c} 2$ cell line may not be as easy to manipulate as previously thought, and their use as a cardiac model in future research should be thoroughly assessed due to their apparent resistance to differentiation.

\section{METHODS AND MATERIALS}

Lab I

\section{Cell culture and general cell maintenance}

An $\mathrm{H} 9 \mathrm{c} 2$ (2-I) cell line, originally derived from embryonic left ventricular rat heart tissue through the method of selective serial passaging, ${ }^{(9)}$ was purchased through Sigma Aldrich from the European Collection of Authenticated Cell Cultures (ECACC), Salisbury, UK. These cells were cultured in Dulbecco's Modified Eagle Medium (DMEM) (Gibco, 41965-039) supplemented with 10\% foetal bovine serum (FBS) (Biocom Biotech, FBS-GI-I2A) and I\% penicillin/streptomycin solution (ThermoFisher Scientific, $15140-122$ ), at $37^{\circ} \mathrm{C}$ under $95 \% \mathrm{O}_{2}$ and $5 \%$ $\mathrm{CO}_{2}$. $\mathrm{H} 9 \mathrm{c} 2$ cells adhered well to the surface of culture dishes and no adhesion factor was necessary. Culture medium was refreshed every second day and the cells were maintained until they reached $70 \%$ confluency. Once confluent, culture medium was discarded and trypsin (Trypsin-EDTA, Gibco, 25200-0I2) was then used to lift the cells from the base of the culture plate. Cells were counted to determine the appropriate seeding densities for each experiment. Experiments were done on cells between passage 7 and 10 .

\section{Differentiation of $\mathrm{H} 9 \mathrm{c} 2$ cells}

$\mathrm{H} 9 \mathrm{c} 2$ cardiomyoblast differentiation was induced according to a protocol described by Pereira, et al. (201 I), in which serum was reduced to $1 \%$ and cells were supplemented daily with I OnM of RA (Sigma, R2625) for II days. ${ }^{(20)}$ For comparison, some cells were maintained in either 10\% FBS (control), or in I\% FBS for the same duration, but without RA.

\section{Morphological assessment}

Morphological changes associated with differentiation of the treated cells were observed using bright field microscopy on days 6 and II of RA treatment. As described by Kimes and Brandt (1976), undifferentiated H9c2 cells appear typically spindle shaped and mononucleated. ${ }^{(9)}$ Upon differentiation however, the cells have been reported to appear as long and thin branched cardiomyotubes. ${ }^{(20)}$

\section{Immunocytochemistry}

H9c2 cells were seeded onto coverslips and grown and treated as previously described. At the end of the treatment protocol, culture medium was removed, cells were washed with ice cold PBS and fixed in cold 4\% paraformaldehyde (Sigma, I58I27) for 10 mins. Cells were washed in cold PBS, blocked in $1 \%$ BSA, permeated with $0.1 \%$ triton solution (Sigma, X-100) and incubated at $4^{\circ} \mathrm{C}$ with cardiac specific troponin $\mathrm{T}$ ( $\mathrm{cTnT}$ ) antibody (Abcam, I ICI I) overnight. The cells on the coverslip were again washed with cold PBS and incubated with an Alexa Fluor anti-mouse secondary antibody (ThermoFisher Scientific, Al l 029) for I hour in the dark. This was followed by staining with Hoechst (ThermoFisher Scientific, HI399), where after 
the slide was left to air dry. Once dry, the coverslip was mounted on a glass slide using Dako fluorescent mounting medium (Diagnostech, S302380) and images were acquired using a Nikon eclipse E400 fluorescent microscope equipped with a DMXI200 CCD Camera 200. Images were acquired using the Nikon plan Flour 10x and 20x objective lens.

\section{Preparation of cell extracts}

Cell extracts were prepared as follows: lysis buffer $(\mathrm{pH} 7.4)$ containing: Tris-HCl (50mM), NP-40 (1\%), Na-Deoxycholate (0.25\%), EDTA (I mM), NaF ( I mM), SBTI $(4 \mathrm{mg} / \mathrm{ml})$, PMSF $(\mathrm{ImM})$, Benzamidine $(I \mathrm{mM})$, leupeptin $(I \mathrm{mg} / \mathrm{ml})$ and Triton $X-100(\mid \mathrm{ml})$ was added to the cells and the cell solution was then transferred to new tubes. Cells were sonicated, centrifuged (8 000rpm at $4^{\circ} \mathrm{C}$ for 10 mins), and the supernatant transferred to new tubes. Protein concentrations were determined with the Bradford method, ${ }^{(22)}$ while others were diluted in Laemmli buffer and stored for Western blot analysis.

\section{Western Blot analysis}

Protein separation was performed on a $12 \%$ stain free, fast cast gel (Bio-rad, 161-0184) which was prepared according to the manufacturer's instructions. The gel was run to completion at $180 \mathrm{mV}$ and then activated on the Chemi Doc ${ }^{\mathrm{TM}}$ XRS system (Bio-rad). The proteins were then transferred onto nitrocellulose membranes (Bio-rad, 170-4156) for 10 mins at 25V and 1.3A. Following transfer, the membrane was blocked for 2 hours in Tris-buffered saline and Tween-20 (TBST, pH7.6) containing $5 \%$ non-fat dry milk and incubated overnight at $4{ }^{\circ} \mathrm{C}$ with the Myosin Light Chain (Cell Signaling, D5IIC) primary antibody solution. The membranes were incubated with the appropriate secondary antibody (Cell Signaling, 70745) at room temperature for I hour followed by washes with TBST. The Pierce ECL Western blotting substrate (Thermo Scientific, 32106) was used to detect protein bands using the ChemiDoc ${ }^{\text {TM }}$ XRS+ System with Image Lab ${ }^{\mathrm{TM}}$ Software (Bio-Rad). The bands generated were expressed using the volume analysis tool and represented as a percentage (\%) of the control sample. GAPDH (Cell Signaling, 1745) was used for the standardisation of protein loading.

\section{Oxygraph analysis}

The assessment of respiration rate is an ideal measure of the oxidative phosphorylation capacity in cells. The Hansatech oxygraph system was used to determine respiration rate in differentiated cells. Assessment was only performed at day 6 for this experiment. The oxygraph was calibrated beforehand according to the manufacturer's protocol. Following treatment, the cells were trypsinized and counted. Then $1 \times 10^{6}$ cells were transferred into the oxygraph respiration chamber containing Hedly's intracellular buffer. Saponin ( I $\mu$ M) (Sigma, 47036) was added to the cells to permeate them where after, mitochondrial complex I substrates, malate $(5 \mu \mathrm{M})$ (Sigma, MI000) and glutamate (I $\mu$ M) (Sigma, GI25I) and the mitochondrial com-

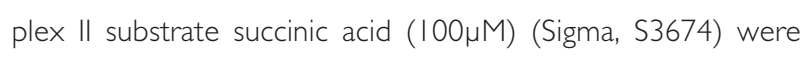
added. Active respiration was induced by the addition of adenosine dinucleotide (ADP) (Sigma, 01905). Respiration rate was then measured for 60 mins. The average respiration rate during this period was determined and compared between groups. Carbonyl cyanide-p-trifluoro-methoxy-phenyl-hydrazone (FCCP) (Sigma, C2920), a mitochondrial un-coupler was used to confirm that mitochondria were actively respiring at the end of the experiment.

\section{Statistical analysis}

All data are presented as the mean \pm SEM. Comparisons between the different groups were performed by one-way ANOVA followed by Bonferroni's post hoc test conducted with the statistical programme GraphPad Prism, version 5.0 (GraphPad Inc.). A value of $p<0.05$ was considered statistically significant.

\section{Lab 2}

\section{Cell culture and general cell maintenance}

The H9c2 (2-I) cell line purchased from ECACC through Sigma Aldrich was maintained in a proliferative state through culture in high (4.5g/L) glucose DMEM (Lonza, BEI2-604F) supplemented with 10\% FBS (Sigma, F6178) and I\% penicillin/ streptomycin (Sigma, $\mathrm{P} 4333$ ) at $37^{\circ} \mathrm{C}$ and $5 \% \mathrm{CO}_{2}$, as described above. No attachment factor was necessary.

Growth medium of the cells was changed every 2 - 3 days and the cells were split in a ratio of 1:3 once they reached $70-80 \%$ confluency. Warm phosphate buffered saline (PBS) was used for all washing steps. A combination of trypsinization and cell scraping was used to lift cells from the base of the culture plates for the purpose of cell division, or collecting cells for Western blotting. Centrifugation at 700rpm for 4 mins at $4^{\circ} \mathrm{C}$ was used when pelleting of cells was necessary. Cells between passage 18 and 25 were used for differentiation experiments.

\section{Differentiation of $\mathrm{H} 9 \mathrm{c} 2$ cells}

Once the cells were $70-80 \%$ confluent, differentiation was initiated by switching to DMEM growth medium supplemented with either $1 \%$ FBS or $1 \%$ Horse Serum (HS) (Biochrome AG, S9135), as well as daily administration of RA (Sigma Aldrich, R2625). Several different concentrations of RA were tested (IOnM, 30nM and I $\mu M$ ). Initially distilled water $\left(\mathrm{d} . \mathrm{H}_{2} \mathrm{O}\right)$ and phosphate buffered saline (PBS) were used for the serialdilution preparation of the RA, however, in subsequent protocol repeats, dimethylsulphoxide (DMSO) (Merck, I02931) was used as RA was found to be inadequately dissolved in the d. $\mathrm{H}_{2} \mathrm{O}$ and PBS. DMSO alone was therefore also used as a vehicle control. Cells were cultured under these conditions for a period of 8 - 12 days with growth medium changed every second day. Control cultures received DMEM growth medium supplemented with 10\% FBS, and medium was also changed every 2 - 3 days. 


\section{Western Blot analysis for markers of cardiac differentiation} Following treatment, cell lysates were prepared using a bullet blender homogeniser (Next Advance Bullet Blender 24) and lysis buffer (200mM Tris+ EGTA, IOmM EDTA, IM NaCl, ImM B-glycerophosphate, $2.5 \mathrm{mM}$ tetra-Na-pirophosphate,

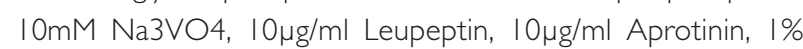
Triton $x-100$ and $50 \mu \mathrm{g} / \mathrm{ml}$ PMSF). Protein determination was carried out using the Bradford method. ${ }^{(22)}$ Following 5 mins of boiling, lysates were stored at $-80^{\circ} \mathrm{C}$ in Laemmli sample buffer. Cardiac tissue samples were also prepared via pulverising and homogenising frozen heart tissue in a lysis buffer solution. These lysates were used as positive controls for cardiac specific markers.

Depending on the protein yield, between 15 - $18 \mu \mathrm{g}$ of protein was loaded per sample and resolved according to the molecular weight on an SDS-polyacrylamide gel electrophoresis (SDSPAGE) system, using a 12\% acrylamide gel. This was followed by transfer of the proteins to an inert polyvinyl fluoride (PVDF) membrane via a wet tank-transfer or semi-wet TurboBlot system. Protein samples were fixed onto the membrane and $5 \%$ milk was used as a blocking agent to minimise non-specific binding of antibodies. The specific proteins of interest were selected by overnight incubation of membranes at $4^{\circ} \mathrm{C}$ with primary antibodies directed against cardiac specific markers, myosin-Light chain 2v (MLC2v) (Cell Signaling, 1297) and Cardiac Troponin T (cTnT) (Cell Signaling, 5593). (20) Membranes were then further incubated at room temperature with antirabbit secondary antibody (Cell Signaling, 7074), and visualised using a chemiluminescence interaction between the detection reagent and the anti-rabbit horseradish peroxidase conjugated secondary antibody. Proteins were detected using either a Chemi-Doc system in association with stain-free technology, ${ }^{(23)}$ or exposure in a traditional dark room. Stain-free technology was used to confirm equal loading (supplementary data).

This procedure was repeated 3 times with between 2 - 4 cell plates per condition (I0nM RA, 30nM RA, I $\mu$ M RA, control, vehicle control and positive heart sample controls).

\section{RESULTS}

Lab I

\section{Morphological assessment}

Differentiation of $\mathrm{H} 9 \mathrm{c} 2$ cells was induced through serum reduction and daily supplementation with RA for II days, as described above. Undifferentiated H9c2 cardiomyoblasts are typically mononucleated and spindle shaped, ${ }^{(9)}$ whereas once differentiated, the cells have been reported to form long, thin, branched multinucleated cardiomyotubes. ${ }^{(20)}$

After 6 days of daily RA treatment and/or serum reduction, only very few cells had begun to display the anticipated cardiomyotube morphology. According to Yang and colleagues
(20/4), immature cardiomyocytes present themselves in a circular (rounded) shape while mature cells are rod (elongated) shaped. (24) When compared to control cells (Figure IE), no obvious differences were observed between the serum starved group (Figure IA) and the serum starved plus RA treatment group (Figure IB). Differentiation treatment was continued and by day I I more cells appeared to have undergone differentiation in both groups when compared to control (Figures IC and ID). These cells presented with elongation and shrunken width indicative of differentiation (supplementary Figures I and 2).

\section{Cardiac troponin $\mathrm{T}$ (cTnT) expression}

Immunocytochemistry was used to assess the expression of cTnT indicative of differentiation. In addition, the number of nuclei per cell were also noted. Cells expressing cTnT, as well as presenting more than 2 nuclei, were regarded as differentiated cardiomyotubes. Figure 2A indicates that after 6 days, serum reduction alone did not result in substantial CTnT expression. However, Figure 2B shows that the combination of serum reduction with RA supplementation did increase the expression of cTnT, when compared to control cells (Figure 2E) after 6 days. By day II, the expression of CTnT in the serum starved group (Figure 2C) had increased in comparison to day 6 (Figure 2A), with more cardiomyotubes containing multiple nuclei. Similarly, the combination of serum reduction and RA resulted in a greater expression of $\mathrm{cTnT}$ after I I days (Figure 2D), and a greater capacity to induce the formation of multinucleated cardiomyotubes. Supplementary Figure 2 shows the morphology of these cells in conjunction with the fluorescent signal.

\section{Myosin Light Chain 2V (MLC-2v) expression}

The expression of a second cardiac specific marker, MLC, was investigated using Western blotting in an effort to further distinguish between myoblasts and differentiated cardiomyotubes. MLC has been used as an indicator of myoblast differentiation into cardiomyotubes. ${ }^{(25)}$

Figure 3 shows that the combination of serum starvation with RA supplementation significantly increased the expression of the marker $(209.5 \pm 7.4 \%, \mathrm{p}<0.00 \mathrm{I})$ when compared to the serum only (1 18.4 $\pm 9.9 \%)$ and control (100.0 $\pm 9.8 \%)$ group on day 6. A substantial increase in the expression of MLC was further observed in both the serum only $(236.9 \pm 16.6 \%$, $p<0.00 \mathrm{I})$ and combination group $(244.4 \pm 9.2 \%, p<0.00 \mathrm{I})$ compared to the control by day II of treatment. Large differences were also observed between the reduced serum groups on days 6 and II. The results obtained here suggest that under conditions of reduced serum only, the expression of cardiac specific markers takes much longer than when serum reduction is combined with RA supplementation. However, it was also noted that RA appears to lose its effect on the expression of MLC when the treatment period is extended. 

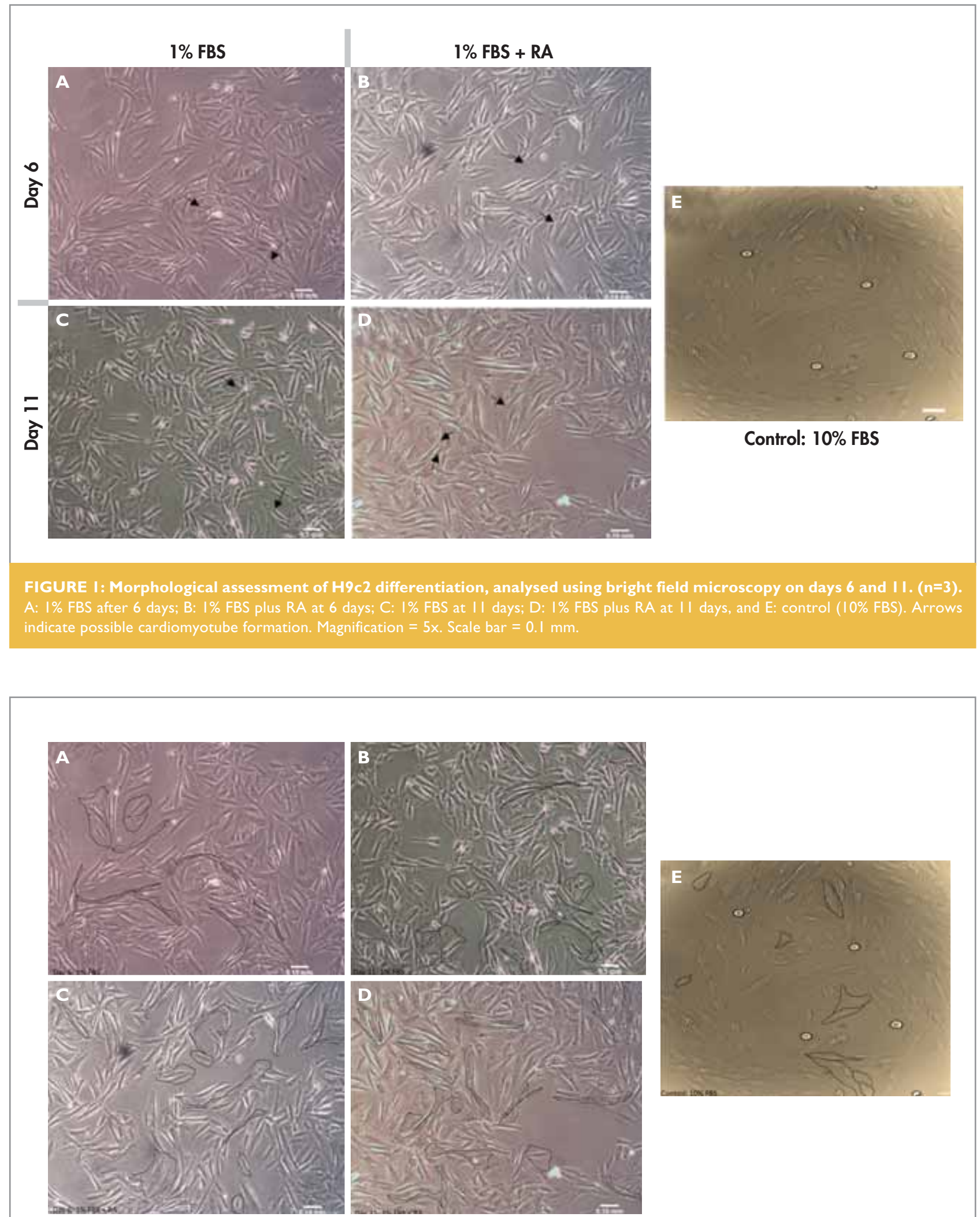

SUPPLEMENTARY FIGURE I: Morphological assessment of H9c2 differentiation, analysed using bright field microscopy on days 6 and II. $(n=3)$. A: I\% FBS after 6 days; B: I\% FBS at II days; C: I\% FBS plus RA at 6 days; D: I\% FBS plus RA at II days, and E: control ( $10 \%$ FBS). Cell outlines indicate differences in morphology attributable to the degree of maturity or differentiation of the cells, where a round appearance is associated with immature cells, while the rod shaped cells are more mature. Magnification $=5 \mathrm{x}$. Scale bar $=0.1 \mathrm{~mm}$. 


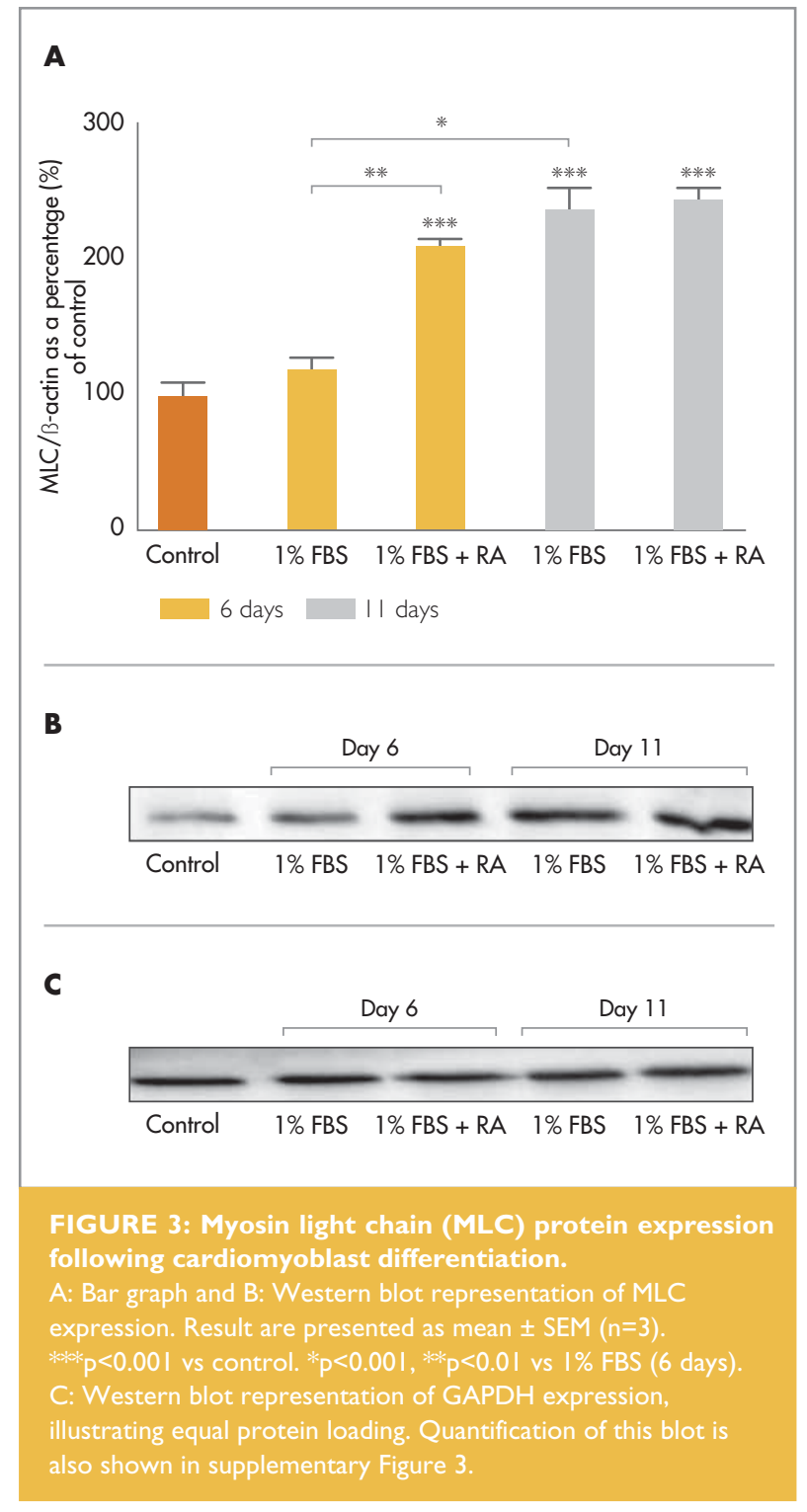

\section{Oxidative phosphorylation analysis}

The switch in preferential energy metabolism, from glycolysis to oxidative phosphorylation, is a fundamental characteristic observed in differentiated cardiac cells. ${ }^{(21)}$ Thus determining the rate of oxygen respiration would provide an ideal measure of oxidative phosphorylation in differentiated $\mathrm{H} 9 \mathrm{c} 2$ cells. It should be noted that this variable was only investigated in cells differentiated for 6 days and no fatty acids were included as the researchers merely aimed to establish if a change in oxygen consumption was evident following differentiation. A significant increase in respiration rate was observed in both serum starved, and serum starved plus RA groups versus the control (100.0 \pm 6. InM $\mathrm{O}_{2}$ /million cells), indicative of differentiation (Figure 4). Large differences were also observed between the reduced serum group $(131.9 \pm 5.2 \mathrm{nM} \quad \mathrm{O} 2 /$ million cells, $p<0.05)$ compared to the serum starved plus RA group (230.7 \pm I 5.8nM $\mathrm{O}_{2}$ /million cells, $\mathrm{p}<0.00 \mathrm{I}$ ).

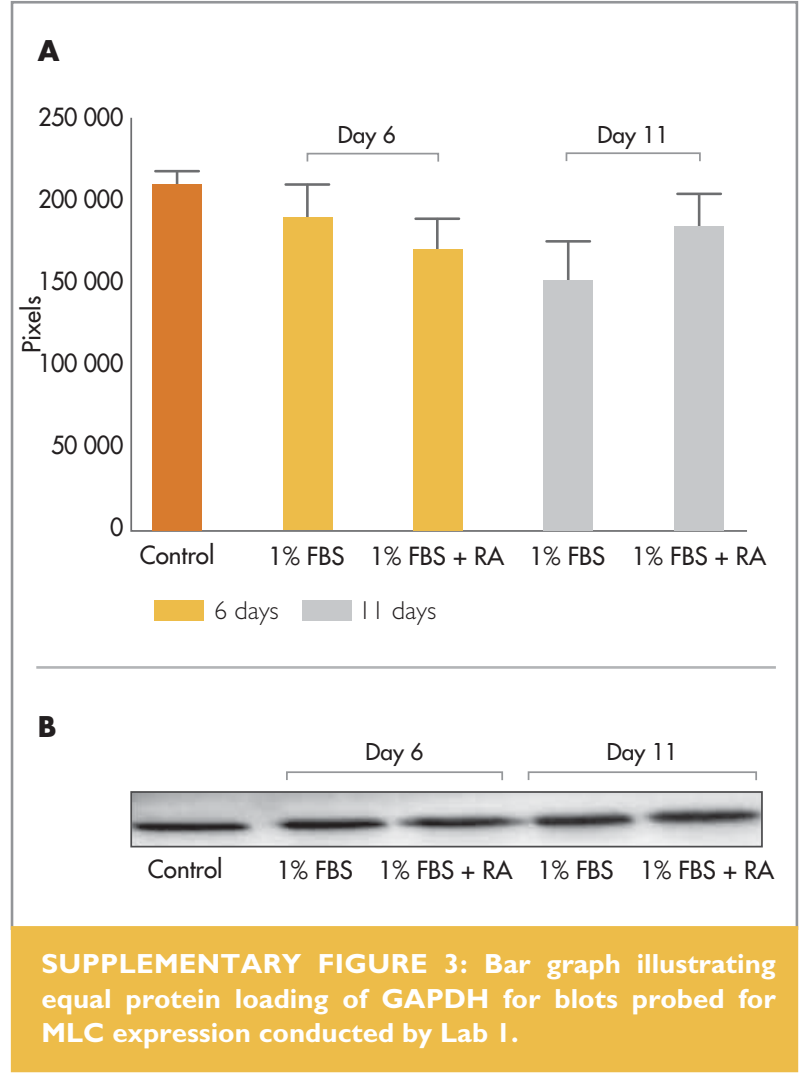

From the above results it can be seen that serum starvation, combined with daily RA supplementation, presents a positive influence on cardiomyoblast differentiation and the expression of cardiac specific proteins. It was also evident that supplementation with RA mediated the switch in substrate utilisation during differentiation to oxidative phosphorylation. Although a reduction in serum concentration was seen to produce similar characteristics, the effects were far less pronounced. This study, however, was not able to establish a definitively homogenous

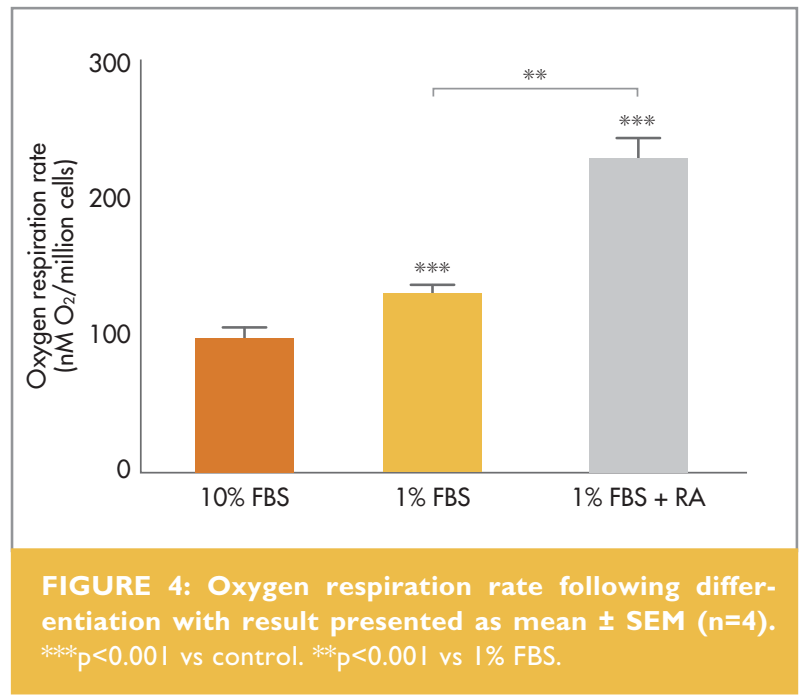


cell population, and even though many cells did undergo differentiation, the majority seemed to remain undifferentiated as was evident from light microscopy.

\section{Lab 2}

In this laboratory, cells were treated daily with RA (I OnM, 30nM and $I \mu M)$ and reduced serum ( $1 \%$ FBS or HS), and cultured over a period of 8 - 12 days, followed by Western blot analysis probing for the cardiac specific markers CTnT and MLC-2v. Figure 5, however, clearly indicates that in our investigations no presence of either of these 2 cardiac markers was detected via Western blotting methods. The only visible bands appeared under the positive heart control samples, suggesting no fault with the primary or secondary antibodies used, or the blotting techniques, and rather indicating that no significant differentiation of the $\mathrm{H} 9 \mathrm{c} 2$ cells had taken place.

This lack of meaningful differentiation was confirmed by initial light microscopy observations (supplementary Figure 5), which revealed a cell population similar to the undifferentiated cell type, with only a few cells showing changes in cell morphology - echoing the results obtained in Lab I.

\section{DISCUSSION}

The $\mathrm{H} 9 \mathrm{c} 2$ cell line is a versatile cell line, which due to its cardiac origin, has been used extensively in cardiac research. The cells are, however, fundamentally undifferentiated myoblasts. It has previously been reported that culturing these cells over a period of days in relatively low concentrations of FBS, as well as in the presence of RA will stimulate differentiation into a more cardiac-like phenotype. We were, however, unable to elicit suitable differentiation as defined by attaining a relatively homogenous cell population. These results were obtained in 2 laboratories working independently of each other, thereby confirming the resistance of these cells to differentiation.

Three different parameters were used to assess the level of differentiation, namely: metabolic profile, expression of cardiac

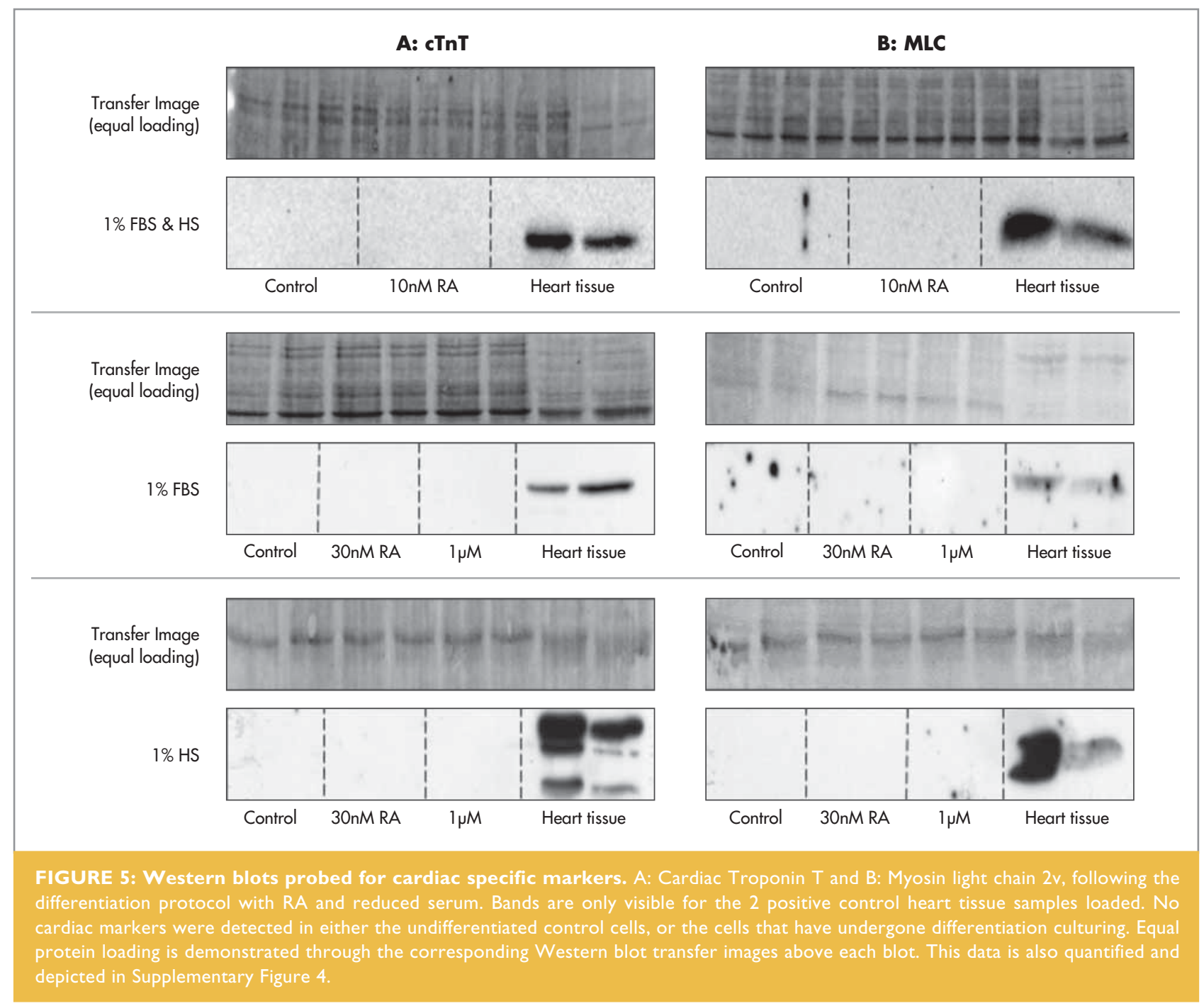




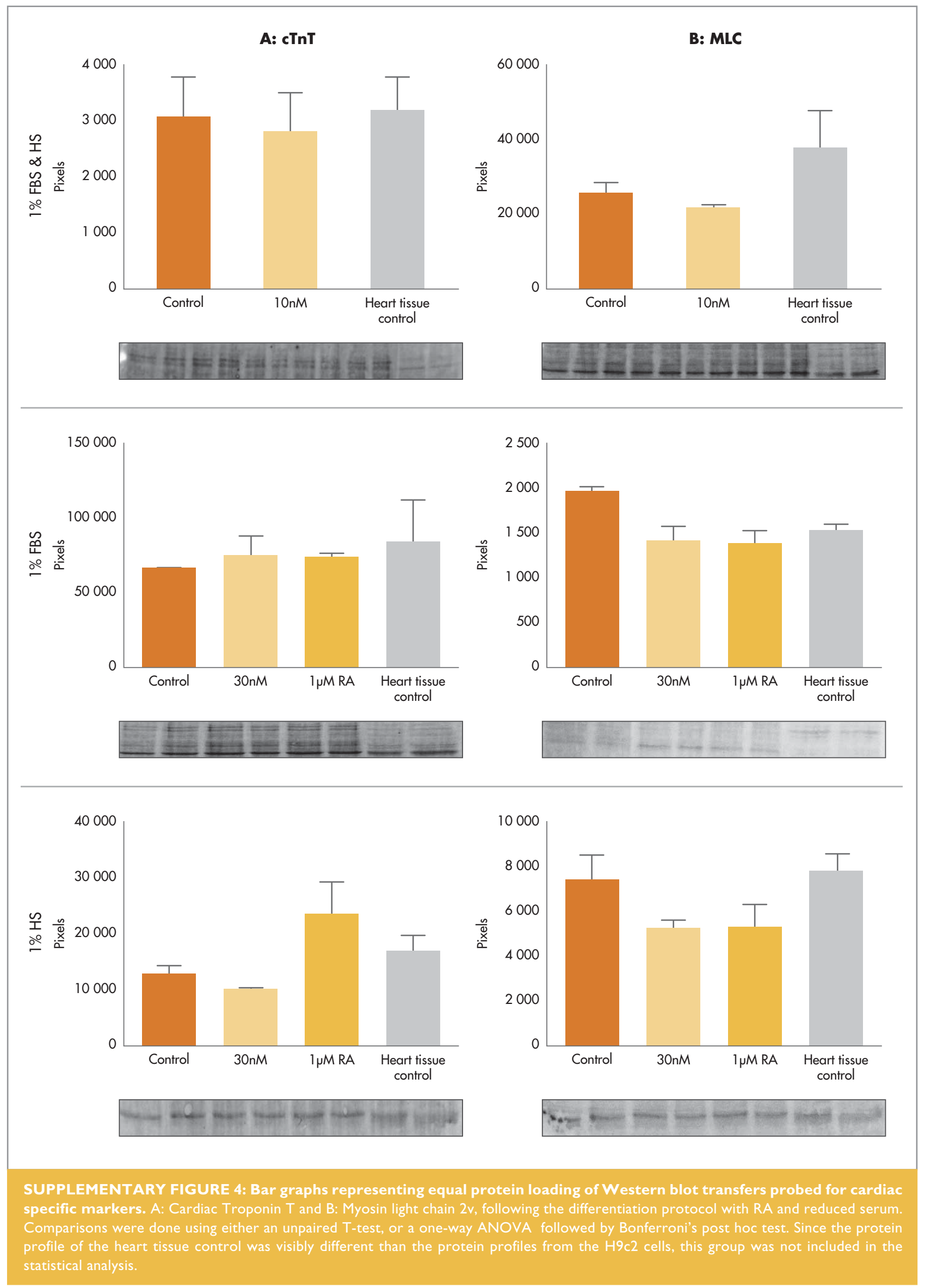



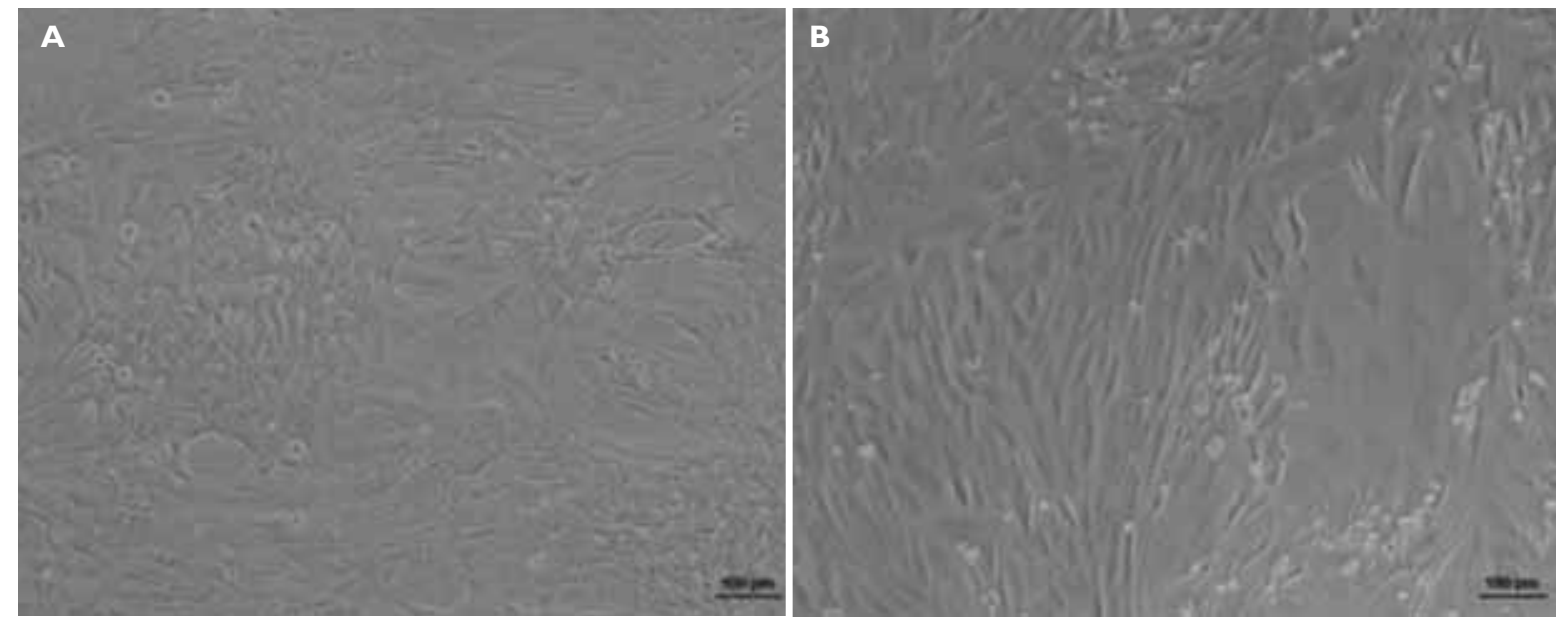

SUPPLEMENTARY FIGURE 5: Representative images of the morphological changes occurring with differentiation in Laboratory 2. A: H9c2 control cells ( $10 \%$ FBS) compared to B: cells exposed to treatment with I $\mu M$ RA and reduced serum (I\% FBS) for a period of 12 days. Some of the cells exposed to treatment showed some signs of morphological change but overall, little differentiation was visible. A Nikon Eclipse TE2000-S Laser Dissection and Fluorescence microscope was used. Magnification = I0x. Scale bar = 100 $\mathrm{mm}$.

specific proteins and cell morphology. Concerning metabolic preference, it is known that heart tissue is predominantly dependent on oxidative phosphorylation, while the H9c2 myoblasts are mostly dependent on glycolytic metabolism. Using the described differentiation protocol, Pereira and colleagues ${ }^{(20)}$ demonstrated a shift from glycolytic metabolism to oxidative phosphorylation in the differentiated cells. We also observed such a shift in terms of an increased capacity for the differentiated cells to utilise oxygen. This was especially true for cells treated with RA. This observation confirms that although long-term culture in $1 \%$ FBS can induce differentiation, ${ }^{(9,18)}$ the addition of RA is necessary to optimally drive the cells in the direction of cardiomyotubes. ${ }^{(20)}$ Investigations into the expression of cardiac specific proteins, specifically $C T n T$ and MCL, revealed a less robust degree of differentiation. Lab I showed an increase in the expression of both these proteins, again dependent on the inclusion of RA in the culturing media, while Lab 2 failed to demonstrate any expression of these proteins, despite testing several concentrations of RA, as well as comparing FBS with horse serum. Finally, cell morphology also indicated a less than optimal result, in that a relatively small proportion of the cell population exposed to the differentiation media demonstrated the expected morphology. As with the other measurements, the inclusion of RA was associated with a greater increase in the degree of the total cell population showing multi-nucleation.

It is interesting that Lab | found quite an impressive degree of differentiation in terms of protein expression and oxygendependent metabolism, which was not necessarily reflected in the degree of morphological change. This could be due to differences in the stage of differentiation, where it could be argued that intracellular changes precede morphological differentiation. Our criteria for the experimental use of differentiated $\mathrm{H} 9 \mathrm{c} 2$ cells was that the cells should be homogenous and differentiation complete, as determined by several end-points. The fact that many of the cells did not have the morphology of mature heart cells therefore led to doubt concerning the exact nature of these cells and if they were completely differentiated. Although the use of several end-points is the optimal way to assess differentiation, it is a short-coming in the field that there are no consensus characteristics and criteria to define differentiated $\mathrm{H} 9 \mathrm{c} 2$ cells. This lack could impede effective comparison and replication in science.

In summary, our combined results indicate that incubation of H9c2 cells for between 6 - 12 days with $1 \%$ FBS and IOnM RA does stimulate differentiation of the cells into a more cardiaclike cell, although this only occurs in a portion of the cells. The protocol is therefore unsuccessful in stimulating robust differentiation capable of generating a homogenous cell population. This therefore limits the potential utilisation of this approach for general research purposes. Potential reasons for the discrepancy between our laboratories and the work published by Menard, et al. ${ }^{(19)}$ and Pereira, et al. (20) are unknown. Two potential confounding variables must be kept in mind, namely (i) the passage number and history of the cells used in the differentiation experiments and (ii) the handling of RA. 
Concerning the passage number, there does not seem to be consensus regarding the optimal passage number for experimenting with $\mathrm{H} 9 \mathrm{c} 2$ cells and, in fact, information concerning the effects of aging cell culture on the cells seems sparse. Recently, Witek and colleagues reported that passage number influences the response of $\mathrm{H} 9 \mathrm{c} 2$ cells to cytotoxic agents. (26) In the context of differentiation, passage number is an unknown, but probably relevant, variable.

Regarding the handling of RA there are 2 important factors to consider: (i) RA is light sensitive and (ii) it is also extremely hydrophobic. The combination of these make it virtually impossible to be absolutely confident that enough RA is truly dissolved in the growth medium to interact with the $\mathrm{H} 9 \mathrm{c} 2$ cells. It has been reported that RA is stable under standard cell culturing conditions and, at least in the context of stem cells, is readily taken up by cells in culture ${ }^{(27-29)}$ with a half-life ranging between $3.5^{(30)}$ and $6^{(31)}$ hours, although 24 hours seems to be an acceptable interval for the replenishment of RA. Both Tsukuda, et al. and Sharrow, et al. reported on the necessity of working with RA in the dark, or under yellow light illumination. ${ }^{(28,29)}$ We took great care to protect the RA from light, while also dissolving it in sequential dilutions of DMSO before adding it to an aqueous medium. The dynamics of RA uptake in $\mathrm{H} 9 \mathrm{c} 2$ cells is however unknown. This is a shortcoming in the existing literature on the use of RA to mediate the differentiation of $\mathrm{H} 9 \mathrm{c} 2$ cells.

Despite our negative results, the benefit of differentiating the $\mathrm{H} 9 \mathrm{c} 2$ cells into a more cardiac-like cell type is without doubt. Potential alternative variations on the current protocol include investigating the addition of other growth factors, in addition to $R A$, in order to drive a more robust differentiation, or finding alternative ways to effectively administer RA. These could include the generation of micelles, or the use of nanoparticles to ensure effective delivery of RA to the cells, or even just the inclusion of albumin in the growth medium in order to stabilise the RA. ${ }^{(28)} A$ third variable to investigate is the time allowed for differentiation. Although both Menard, et al. ${ }^{(19)}$ and Pereira, et al. ${ }^{(20)}$ cultured their cells for 5 - 7 days, it is noteworthy that for the original description of the $\mathrm{H} 9 \mathrm{c} 2$ cells by Kimes and Brandt ${ }^{(9)}$ cells started showing pronounced myotube formation after 15 days of culturing and much less so after 7 days. It is therefore conceivable that an extended period of culturing, in the range of 15 - 20 days, might produce a more pronounced degree of differentiation. Yet a fourth variable, which has received some attention, is the surface on which the cells are cultured and differentiated. In this regard it has been reported that the degree of differentiation is influenced by interventions such as growing cells on an extracellular matrix ${ }^{(32)}$ or growing cells on a surface with micro grooves. ${ }^{(33)}$

The most significant limitation of this study is that it was done in a retrospective manner, in other words the research efforts of the 2 laboratories were not planned or coordinated in advance. Effective collaboration would have entailed measuring the same end-points, making use of the same protocols and exchanging cells between laboratories at different points in the protocol. This limitation is however also a strength, since despite the absence of collaboration, we still reached similar conclusions.

In conclusion, long-term culture of $\mathrm{H} 9 \mathrm{c} 2$ cells with $1 \% \mathrm{FBS}$ and RA could induce a degree of differentiation, although this seemed limited to certain cells. The absence of a homogenous differentiated cell population, however, complicates the use of these cells for research. Investigators thus need to be aware of this when selecting this as an experimental model for their research.

\section{ACKNOWLEDGEMENTS}

The financial assistance of the National Research Foundation (NRF), and the South African Medical Research Council (SAMRC) under a self-initiated grant, towards this research is hereby acknowledged. Opinions expressed and conclusions arrived at, are those of the author and are not necessarily to be attributed to the NRF or the SAMRC.

Conflict of Interest: The authors declare no conflict of interest and all authors have approved the final manuscript submitted. 
I. Beaglehole R, Yach D. Globalisation and the prevention and control of non-communicable disease: The neglected chronic diseases of adults. The Lancet 2003;362(9387):903-908.

2. World Health Organisation (WHO). Cardiovascular diseases (CVDs). 2015 (online) Retrieved March 10, 2016: http://www.who.int/mediacentre/ factsheets/fs $317 /$ en/.

3. Sliwa K, Wilkinson D, Hansen C, et al. Spectrum of heart disease and risk factors in a black urban population in South Africa (the Heart of Soweto Study): A cohort study. The Lancet 2008;37। (9616):915-922.

4. Onen CL. Epidemiology of ischaemic heart disease in sub-Saharan Africa. Cardiovasc J Afr 2013;24(2):34-42.

5. Bradshaw D, Groenewald P, Laubscher R, et al. Initial burden of disease estimates for South Africa, 2000. South African Medical Journal 2003: 93(9):682-688.

6. Jamison DT. Disease and mortality in sub-Saharan Africa. World Bank Publications; 2006.

7. Steyn K, Fourie JM. The heart and stroke foundation South Africa. Media Data Document.Cape Town: Department of Medicine, University of Cape Town, and Chronic Diseases of Lifestyle Unit 2007.

8. Chaudry A. Cell Culture. 2004 (online). Retrieved April 12, 2016: http:// www.scq.ubc.ca/cell-culture/.

9. Kimes B, Brandt B. Properties of a clonal muscle cell line from rat heart. Exp Cell Res 1976;98(2):367-381.

10. Hescheler J, Meyer R, Plant S, et al. Morphological, biochemical, and electrophysiological characterisation of a clonal cell ( $\mathrm{H} 9 \mathrm{c} 2)$ line from rat heart. Circ Res 1991;69(6):|476-| 486.

II. Sipido KR, Marban E. L-type calcium channels, potassium channels, and novel nonspecific cation channels in a clonal muscle cell line derived from embryonic rat ventricle. Circ Res 1991;69(6): I 487-1499.

12. Watkins SJ, Borthwick GM, Arthur HM. The H9C2 cell line and primary neonatal cardiomyocyte cells show similar hypertrophic responses in vitro. In vitro cellular \& developmental biology-animal 20 I 1;47(2): | 25- 131 .

13. Chou $H$, Chen $Y$, Lee $T$, et al. Proteomics study of oxidative stress and Src kinase inhibition in $\mathrm{H} 9 \mathrm{C} 2$ cardiomyocytes: A cell model of heart ischaemia reperfusion injury and treatment. Free Radical Biology and Medicine 20 I0; 49(1):96-108.

14. Turner NA, Xia F, Azhar G, et al. Oxidative stress induces DNA fragmentation and caspase activation via the c-Jun $\mathrm{NH}$ 2-terminal kinase pathway in H9c2 cardiac muscle cells. J Mol Cell Cardiol 1998;30(9): 1789-1801.

15. Jennings RB. Historical perspective on the pathology of myocardial ischaemia/ reperfusion injury. Circ Res 20 13; 1 13(4):428-438.

16. Yellon DM, Hausenloy DJ. Myocardial reperfusion injury. N Engl J Med 2007;357( ( I): 1 121-1 1 35 .

17. Sishi BJ, Loos B, van Rooyen J, et al. Doxorubicin induces protein ubiquitination and inhibits proteasome activity during cardiotoxicity. Toxicology 2013;309:23-29.

18. Pagano M, Naviglio S, Spina A, et al. Differentiation of H9c2 cardiomyoblasts: The role of adenylate cyclase system. J Cell Physiol 2004;198(3):408-4I6.

19. Menard C, Pupier S, Mornet D, et al. Modulation of L-type calcium channe expression during retinoic acid-induced differentiation of $\mathrm{H} 9 \mathrm{C} 2$ cardiac cells. J Biol Chem 19998;274(4I):29063-29070.

20. Pereira SL, Ramalho-Santos J, Branco AF, et al. Metabolic remodeling during $\mathrm{H} 9 \mathrm{c} 2$ myoblast differentiation: Relevance for in vitro toxicity studies. Cardiovascular toxicology 201 I; I (2): 180-190

21. Lopaschuk GD, Jaswal JS. Energy metabolic phenotype of the cardiomyocyte during development, differentiation, and postnatal maturation. J Cardiovasc Pharmacol 2010 Aug;56(2): I30- 140.

22. Bradford MM. A rapid and sensitive method for the quantitation of microgram quantities of protein utilising the principle of protein-dye binding. Anal Biochem 1976;72:248-254.

23. BioRad. ChemiDoc MP System. 2015. (online). Retrieved March II, 20I5: http://www.bio-rad.com/en-us/product/chemidoc-imaging-systems/ chemidoc-mp-system.
24. Yang $X$, Pabon L. Murry CE. Engineering adolescence: Maturation of human pluripotent stem cell-derived cardiomyocytes. Circ Res 2014; I | 4(3):5 I I-523.

25. Comelli M, Domenis R, Bisetto E, et al. Cardiac differentiation promotes mitochondria development and ameliorates oxidative capacity in $\mathrm{H} 9 \mathrm{c} 2$ cardiomyoblasts. Mitochondrion 201 I; I I (2):315-326

26. Witek $P$, Korga A, Burdan F, et al. The effect of a number of $\mathrm{H} 9 \mathrm{C} 2$ rat cardiomyocytes passage on repeatability of cytotoxicity study results. Cytotechnology 2016:1-9.

27. Gubler ML, Sherman MI. Metabolism of retinoids by embryonal carcinoma cells. J Biol Chem 1985;260( 17):9552-9558.

28. Tsukada M, Schröder M, Seltmann H, et al. High albumin levels restrict the kinetics of 13 -cis retinoic acid uptake and intracellular isomerisation to alltrans retinoic acid and inhibit its anti-proliferative effect on SZ95 sebocytes. J Invest Dermatol 2002; | 19(1): 182-185.

29. Sharow KA, Temkin B, Asson-Batres MA. Retinoic acid stability in stem cell cultures. Int J Dev Biol 2012;56(4):273-278.

30. Williams JB, Napoli JL. Metabolism of retinoic acid and retinol during differentiation of F9 embryonal carcinoma cells. Proc Natl Acad Sci USA 1985;82(14):4658-4662.

31. Redfern CP, Todd C. The effects of retinoic acid on rat epidermal cells in vitro: changes in patterns of protein phosphorylation in relation to growth and differentiation. Exp Cell Res 1988; 174(2):367-377.

32. Suhaeri M, Subbiah R, Van SY, et al. Cardiomyoblast (H9c2) differentiation on tunable extracellular matrix microenvironment. Tissue Engineering Part A 2015;21(11-12):1940-1951.

33. Anene-Nzelu CG, Choudhury D, Li H, et al. Scalable cell alignment on optical media substrates. Biomaterials 2013;34(21):5078-5087. 